\title{
An Ideal Model to Measure Humorousness: 1+1 Two Factor Formulae
}

\author{
Wanfang Zhang \\ School of Foreign Languages, Wuhan Polytechnic University, Wuhan, China
}

\begin{abstract}
At present, humor is mainly studied on its internal mechanism and communication function. Humorousness is an indicator of humor quality, usually equivalent to the comic effect generated by the verbal humor in a special context. It is not a scattered random sequence that can be qualitatively defined only, but measurable and adjustable through a set of pragmatic devices and strategies. Based on Relevance Theory, this paper is intended to establish an ideal model "1+1 Two Factor Formulae" to quantify humorousness of verbal humor by deconstructing traditional Incongruity-Resolution Theory. It is expected to shed some light on humor research and give some inspiration to stylists and humorous writers.
\end{abstract}

Index Terms-humorousness measurement, incongruity, resolution, relevance theory

\section{INTRODUCTION}

Humor research can be traced back to the time of Aristotle and Plato from different disciplines involving philosophy, aesthetics, psychology, sociology, anthropology and linguistics. There are three main traditional humor theories to explain humor: Superiority Theory, Relief Theory and Incongruity Theory.

Humorousness is an indicator to test the quality of humor, generally equivalent to the comic contextual effects achieved by verbal humor in certain contexts. Humorousness is measurable because it is an indirect speech act, and the indirectness of language directly influences the contextual effects of language, which can be measured by its "relative length" through the manifestation of the "degree of its zigzag line" (Hou Guojin, 2004). Therefore, it is reasonable to believe the feasibility of humorousness measurement. This paper develops a set of " $1+1$ two factor formulae" to bridge humorousness with the degree of relevance on the basis of the traditional incongruity - resolution theory.

\section{II. $1+1$ Two FACTOR FormULAE}

Prototype and Function of $1+1$ two Factor Model

" $1+1$ two Factor" Model is aspired by the traditional theory of humor research: Incongruity - Resolution. According to this theory, the process of interpretation of humor is roughly deconstructed into two sub-processes: the interpretation of incongruity, and the interpretation of resolution. Although not a clear time boundary has been made between the two sub-processes, and even some think they are ongoing simultaneously (because the hearer almost begins to make response, either laughing or humming, the very second he senses the incongruous part in the story), they are desperately two independent components for the hearer (the addressee) to deal with: the former is tangible and physical, for incongruity can be sensed by the hearer's common sense, and the latter is intangible and mental, which depends on the hearer's cognition and inferential capacity, and to the largest extent, on whether the inference results are amusing enough to bring about laughter. A joke maker mostly fails in a cold joke as the incongruous elements in his story is only captured but not appreciated by his addressee, so the process of resolution seems weightier for success of verbal humor. Based on this belief, resolution is completely independent from incongruity in this research, which outlines the prototype of our model:

\section{Incongruity + Resolution $=$ Humor}

(1) $+(\mathbf{1})=(?)$

The model " $1+1$ " indicates the interaction of two independent factors: incongruity and resolution, and their interaction affecting the change of humorousness. Considering the number of incongruity and resolution, and for mathematics" sake, we coin two terms "incongruitum" and "resolutor" to replace the above two in the prototype model, and convert humor into humorousness to make the equation more direct and illustrative, so the final model is:

\section{Incongruitum + Resolutor $=$ Humorousness}

(1) $+(\mathbf{1})=(?)$

The model appears simple and easy to operate, however, measurement of humorousness is too complicated to be covered by the interaction of merely two factors. Other variables or factors are probably involved including the hearer's cognitive ability, emotion, culture differences, and contexts. Only cognitive ability may cover various factors like gender, age, job occupation, area etc. If so, this task will be an impossible mission, so it is just a basic yet ideal model by which major factors or variables affecting humorousness are taken into account. That is to say, "interferon" is 
possibly excluded from this model, leaving only three basic parameters and two factors as said above, and it targets at simple verbal humor only for a preliminary test of its validity.

1) Settings of three Major Parameters

Tan Daren's “a piece of comic work generated by the author with a sense of comic spirit for the special purpose (1997, p. 5)" plus Kerbrat-Orechioni's pragmatic supplementation "of whose perlocutionary (intended) effect is laughter" is selected in this paper as the definition of humor (to be exact, verbal humor) because this one is the plain reflection of the three major attributes of verbal humor, which will be used as the three parameters for quantitative analysis.

a) Parameter I: Cognitive Capacity

"Humor is an attribute indispensable to man's welfare" (Xu Lixin, 2004, p. 14); "Humanity is the only species with a sense of humor" (Palmer, 1994, p. 1). Verbal humor is man's works. Talking humor is totally nonsense for lack of the author or the joke maker.

Freud distinguishes three kinds of participants in a successful joke: the addresser, the laughing stock, and the addressee (Wang Yong, 2002). For the addresser and the addressee, there are different synonyms: the author and the audience in rhetoric and the speaker and the hearer from the conversational perspective. However, the relationship between the three kinds of participants in a piece of humor cannot be separated completely. Overlapping and confusion often occur.

The addresser is the humor producer; the laughing stock is the object to be satirized or made fun of, and the addressee is the humor receiver who is motivated to interpret the addresser's comic thought from context. All of the three can be set in or out of the context. Generally, the addressor will keep himself and his addressee away from the context with a common target at the laughing stock, so this humor is to be appreciated by both the addresser and the addressee, called "inclusiveness". If either the speaker or the addressee, or both were placed in the context, the case would become complicated. Not only the role of the addresser might overlap that of the addressee, or the role of the addressee might overlap that of the laughing stock, but the addresser can act the role of all-in-one as shown in the words of the famous cartoon star, the cat of Garfield:

Фa. Money is not everything. There's Master Card \& Visa.

$\Phi$ b. One should love animals. They are so tasty.

$\Phi c$. The wise never marry, and when they marry they become otherwise.

$\Phi d$. Hard work never killed anybody. But why take the risk?

Ф. Work fascinates me. I can look at it for hours!

Hence there are humor participants like Garfield or other creatures likewise; the law that only man creates humor may be vetoed. However, Garfield or those creatures are all personified actors in literary works. After all, those comics are "generated by the author".

The underlined meaning of "an attribute indispensable to man's welfare" for linguists is that humor is subject to man's cognitive capacity. That is, verbal humor should be limited to the cognitive scope of both the author and the addressee; otherwise the estimated effects will fail. In our questionnaire, an English brain teaser is specially designed for the illiterate or half-literate (middle school students or middle aged men with elementary education). Undoubtedly it makes nonsense for the word meaning cannot be comprehended. This is a typical case to prove the failure of humor caused by cognitive incompetence and further confirms our belief that cognitive capacity, also called "cognitive environment" by Sperber and Wilson, is the foundation of verbal humor, the primary parameter to measure humorousness, recorded as Parameter I (P1 for short) hereafter. But for P1, humor is deemed to abortion at the very beginning.

b) Parameter II: Laughing Index

Palmer (1994, p. 3) echoes the "perlocutionary (intended) effect" of humor by the assertion that humor is "everything that is actually or potentially funny and humor is also the process by which this 'funniness' occurs" (Xu Lixin, 2004, pp.17-18). Laughter (chuckle or giggle) is the criterion to appraise the quality of verbal humor in daily life. To be scientific, we term this criteria Laughing Index, the second parameter (P2) to test humorousness.

Palmer's view again resonates incongruity resolution theory in that they both agree that humor is the integral of two laughing indicators: one is the laughing stock "actually or potentially funny" (obviously it should be incongruous in some aspects) and the process of resolution "the process by which this 'funniness' occurs". In a word, the relationship and interaction between those two indicators directly affect the quality of humor. To quantify the degree of humor requires quantification of both two indicators in advance and foundation equation accordingly. However, a single equation cannot solve all the problems as both indicators are changing with a dynamic interaction, so more efforts are made to consider various situations as much as possible, achieving a set of formulae to take an overall account to the most potential.

Laughing index as P2 is the drive and the kernel parameter of humorousness, the focus of our discussion. For the sake of unification and brevity, the two indicators of P2 are converted into two statistical factors: incongruity and resolutor.

c) Parameter III: Context 
The degree of verbal humor changes every minute. Language is the direct or indirect carrier of most jokes or loose talk, which distinguishes them from non-verbal humor like pantomime and antics. Verbal humor is confined to time and space, culture, social communication at its birth and all those are subject to context. Context as Parameter III (P3) plays an important role in measurement of humorousness. To be specific, the change of context leads to the change of the interaction between incongruitum and resolutor. A joker succeeds in placing his intrigue in a proper context, where all the participants and every objective and subjective conditions adapt to the current communicative requirements.

2) Settings of two Factors

Parameter II is subdivided into two indicators: incongruitum and resolutor, the two factors serving as the drive and final destination of verbal humor. Both two factors are derived from the original psychological research on humor by incongruity - resolution proposed by Peplcello (1983). Traditional incongruity - resolution theory stands on the perspective of cognition science, fully demonstrating P2, and commencing P2. An offset of involvement in P3 will remarkably improve the incongruity - resolution theory, nevertheless, most types of verbal humor are still in its domain.

a) Incongruitum

The first concern of theoreticians of the incongruity-resolution school is incongruity. Bergson holds that if one's morality should have been the audience's concern, and yet his physical side catches most attention, the man will be a comic. Bergson stands fro superiority theory, but some of his views mirror incongruity. He attributes "when something mechanical is encrusted on something that is living" to the cause of laugh, with the basic thought of incongruity embedded beneath: the major task of humor is to settle down the differences and disharmony between the expected value and the actual value and incongruity lies in the limitless possibilities hidden behind a series of synonyms like "disagreement", "discord", or "disharmony" (Xu, 2004, p. 25). Each entity or occurrence of such "disagreement", "discord", and "disharmony" is termed as a point of incongruity - an incongruitum as mentioned above.

\section{b) 2.3.2 Resolutor}

Norrick describes humor based on frame shift theory by asserting that "a joke activated a schema and then twisted it", and "interpretation of humor requires first identification of this frame, and rebuild (recomprehend) this frame when encountering information clashed with this frame of expected results" (Liu Naishi, 2005). The rebuilding (recomprehension) process is the process of resolution of an incongruitum.

During the process of resolution, a non-demonstrative inference is ongoing. Unlike logical demonstrative inference as Aristotle's Syllogism, non-demonstrative inference, based on the hearer's encyclopedia knowledge, is a random and fuzzy inference with a probable but not absolute result. The frequency of such a non-demonstrative inference in the process of resolution is the second factor of P2, termed resolutor hereunder. In other words, the frequency of inferences in the recomprehension process is equivalent to the number of resolutors.

Incongruity - Resolution Theory has its domain in that it succeeds in finding the foundation and the drive force of laughter, but it is too theoretical to put into practice for verbal humor fails to be set and reconstructed in a particular context. RT is proposed to offset incongruity theory in this respect. Non-demonstrative inference of RT will serve as the tie to unify three major parameters and two individual factors in a whole.

\section{3) Formation of 1+1 Two Factor Formulae}

With all parameters and factors settled down, the next and most important step to formulate equation is to find out the correlation between parameters and factors, from where RT is cut into this model.

From the above analysis for setting parameters and factors, a conclusion can be drawn that incongruity is the necessary and sufficient condition to generate humor. The existence of one incongruitum is enough to make fun, in need of no resolution. However, pure fun brought by incongruitum(s) takes up quite a little, mainly centered among nonverbal humor. Most jokes and punch lines are generated out of a certain context, which requires the addressee to pay processing efforts for the optimal relevance and obtain the most amount of humorousness. It is easy to figure out that incongruity corresponds to the ostensive stimuli, and resolution is the addressee's ostensive inferences in the process of recomprehension. Plus our pragmatic definition of humorousness, RT is naturally bridged with incongruity-resolution.

According to RT, the value of measured humorousness should have coped with such a law: more relevant, less processing effort and more amount of humorousness (because humorousness is the contextual effects in a piece of verbal humor). By contrast, more irrelevant utterances mean more processing effort and less amount of humorousness. However, the final findings by handing out 300 questionnaires to 3 groups of objects show an opposite result: usually an irrelevant context brings about more pleasure, although the addressee will spend more processing efforts to interpret the context. In other words, the correlation between relevance and humorousness turns to be an irregular or even opposite change against the correlation between relevance and contextual effects of common conversation. After data processing from and analysis of 300 results (over 200 valid ones), our experiment on the correlation between relevance and humorousness yield 4 outcomes, where an incongruitum is recorded as $\mathrm{x}$, a resolutor as $\mathrm{y}$, with unit as 1 , the 4 outcomes are converted into our model to measure humorousness of verbal humor:

Incongruitum(s) + Resolutor $(s)=$ Humorousness 


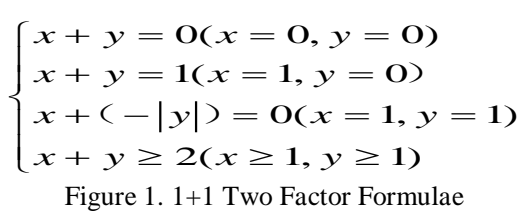

In the set of 1+1 Two Factor Formulae, each factor is assigned with 4 values and each value should be an integer equal or exceeding zero. By restoring those values, the whole set of formulae corresponding to 4 outcomes of our experiment can be converted into the following table.

TABLE 1.

CONVERSION TABLE OF $1+1$ TWO FACTOR FORMULAE

\begin{tabular}{|c|c|c|c|c|c|}
\hline $\begin{array}{l}\text { Incongruitum } \\
(\text { Variable } x)\end{array}$ & $\begin{array}{l}\text { Resolutor } \\
(\text { Variable } y)\end{array}$ & $\begin{array}{l}\text { Humorousness } \\
\text { (Sum) }\end{array}$ & $\begin{array}{l}\text { Contextual } \\
\text { Effect }\end{array}$ & $\begin{array}{l}\text { Processing } \\
\text { Effort }\end{array}$ & Relevance \\
\hline 0 & 0 & 0 & None & None & Max. \\
\hline 1 & 0 & 1 & Max. & None & Max. \\
\hline 1 & $(-) \geqq 1$ & $\leqq 0$ & Negative Growth & Ascend & None \\
\hline$\geqq 1$ & $\geqq 1$ & $\geqq 2$ & Positive Growth & Ascend & Descend \\
\hline
\end{tabular}

Note: neither incongruitum nor resolutor will disappear automatically whether ideal contextual effects are achieved or not, so neither $x$ nor $y$ will be a negative number.

The above table mainly indicates that:

- Zero incongruitum or resolutor $(x=0, y=0)$ means no ostensive inference. There is no need of processing effort for resolution of an unexisting incongruitum. A general ostensive inference for utterance relevance will directly yield the explicit or implicit meaning of this utterance. The utterance is relevant to the largest extent, but there is no contextual effect, so humorousness $(\mathrm{x}+\mathrm{y})$ is zero.

Q. A: Is it raining?

B: Take an umbrella with you.

Ostensive inference according to RT:

When it is raining, people need an umbrella (implicated premise)

B needs to take an umbrella (explicated premise)

It is raining (implicated conclusion 1)

It is going to rain (implicated conclusion 2)

This example illustrates the fuzziness of language. B's answer may yield more than one interpretation. His answer is relevant to A's question to some extent but less relevant than plain reply like "Yes, it's raining". And this conversation contains no "disharmony" or "disagreement". A lack of an incongruitum and a resolutor keeps it out of the domain of verbal humor.

- An incongruitum $(x=1)$ is an ostensive stimulus. Triggered by an incongruitum, contextual effects of humor can be directly achieved even sparing processing effort for resolution $(y=0)$. Typical examples are non-verbal humor like pantomime or antics. In this kind of humor, the audience's reaction is to the large extent relevant to actor's performance, and theoretically, a maximum of contextual effects can be achieved while the humorousness reaches the peak.

Nerhardt (1976) holds that humor is generated from the opposition of two kinds of psychological representation, and the degree of humor is depending on the distance of expected results from its stimuli in a comic matter (Liu Ping, 2005). He designed an experiment to let participants lift a row of weights by order. All those weights look the same, so one cannot judge their weight by eye test. Actually all those weights except the last one are arranged in an order from light to heavy, but the last one is the lightest. Most participants chortled when lifting the last one because of unexpected results. Besides, larger weight variation causes more incongruous effects, so one is more likely to burst into laughter. Nerhardt's experiment is a typical example to prove that a single incongruitum without resolution is enough to generate humorous effects. However, without the process of resolution, no resolutor is generated, nor is a new incongruitum. Such humor is at the risk of "crib death" after being appreciated once. We believe that those participants in Nerhardt's experiment seldom have interests to repeat this game and the fun arising out of the distance between the conspiracy and their expectation will fade away if they are informed of the truth and forced to play it again. From this perspective and in fact, verbal humor requiring resolution is more funny and valuable for academic research.

- Co-existence of incongruitum(s) or resolutor(s) $(x>0, y>0)$ will lead to two opposite tendencies in terms of the growth of humorousness: negative and positive.

- Negative growth of humorousness under the co-existence of incongruitum and resolutor is abnormal. As existed incongruitum resolutors will not disappear under any circumstances, the cause for the loss of humorousness shall be sought from other perspective. Probably, the influence of P1, or P2 or both surpass the role of both factors of P2, serving as the key parameter(s) to write down humorousness. The English brain teaser mentioned in the illustration of P1 is such a case. In a particular context, the addressor's design exceeds the capacity of the addressee's cognition. It is already a communication failure in nature, to say nothing of relevance of humorousness. At the same time, communication failure produces incorrect and negative ostensive inference and incorrect resolutors accordingly. As 
resolutors cannot disappear, "-" in the third equation of the formulae does not mean loss or diminishing of resolutors, but incorrect resolutors which will cause loss of humorousness; meanwhile, "ฏ”does not simply means that humorousness descends down to zero or negative. Humorousness as an indicator to describe quality of humor can never be a negative number. So " $\leqq$ " here indicates that communication failure not only leads to abortion of fun $(=0)$, but also brings about passive consequences like inferiority or revenge in the addressee's mind $(<0)$, for example, if the addressee resembles the laughing stock in the story said by the addressor, the addressee would feel offended even though it is not the addressor's original intention.

P1 and P3 are to the largest extent interacted. Any of the following attributes, including but not limited age, area, gender, social distance, cultural background, education level, and living standards will produce pragmatic failure. For example, adult jokes popular in male employers will insult female colleagues in the same office. Journey to the West (Da Hua Xi You), the masterpiece of Hong Kong's king of comedy, Stephen Chow (Zhou Xingchi), is one of the compulsory courses for student of folk literature of Peking University on the one hand, and on the other hand, his "Wulitou" style of performance is censored by orthodox films. And a story of the dumb or the blind is improper to disabled people anytime.

To be strict, the third equation shall be deleted from our model because it exceeds the range of P2. P2 works for successful communication only. Failure of verbal humor arising out of P1, or P3 is failure of communication.

- The co-existence of incongruitum and resolutor will increase the degree of humor at a speed faster than " $1+1$ ". More surprisingly, the relevance of utterance in verbal humor increases with a reciprocal ratio to that of humorous contextual effects (see Table 1). This is contradictory to RT.

The final inequation of the formulae indicates that the correlation between humorousness and two factors are not simple " $1+1=2$ ". The two factors are mutually stimulated and promoted. Resolution of one incongruitum will generate a new incongruitum and a new resolutor accordingly. Before verifying this hypothesis in the next section and for brevity's sake, we term the first incongruitum "meta-incongruitum", recorded as $n_{0}$, and derived incongruitum(s) in the process of resolution, or re-resolution "neo-incongruitum", recorded $n_{1}, n_{2}, n_{3} \ldots$ As for the factor of resolutor, the first thing is to distinguish inference in the resolution process from that of conventional inference, so we term the inference of conventional inference $r_{0}$. Relatively, the first resolutor is termed initial resolutor for $r_{l}$, and the following resolutors are multi-resolutors $r_{2}, r_{3}, r_{4} \ldots$

\section{Humorousness MeAsurement of Simple Verbal Humor}

From this section, the major task is to verify the validity of our model by case studies. As $1+1$ Two Factor formulae are construed on an ideal model, the cases we choose are possibly simple and typical, which enables us to give a rough description of the growth of humorousness. The process goes from simple to complex (relatively) in order. As the first three equations are illustrated in the last section, this section focuses on the last inequation.

\section{A. Single Incongruitum and Single Resolutor}

The cut-in is the simplest verbal humor with a single incongruitum and a single resolutor.

"A comic text is generally divided into two parts: 'preface' and 'punch line"” (Liu Ping, 2005). According to incongruity-resolution theory, the object reflection of incongruity is the contrast between the actual value and the expected value of the hearer after the occurrence of the punch line. Then governed by RT, one gets used to seeking for the optimal relevance adapting to convention. When plausible optimal relevance is located, the hearer will stop his inference. Therefore, a joker takes advantage by "properly making use of this routine cognitive tendency of the hearer and designing a tactic strategy that the preface is 'misleading, while the punch line is the 'steering wheel' to pull the loser back to the correct way of interpretation."(ibid.) The preface is the foundation of generating an incongruitum, which requires the hearer to make a conventional inference to obtain primary optimal relevance, recorded as $a_{1}$. The punch line is the essence of verbal humor, which requires the hearer to limp out of convention and make a second inference to obtain secondary optimal relevance $a_{2}$. To sum up, the total optimal relevance of verbal humor is the difference between primary optimal relevance and secondary optimal relevance $\left|a_{2}-a_{1}\right|$ (see Figure 2).

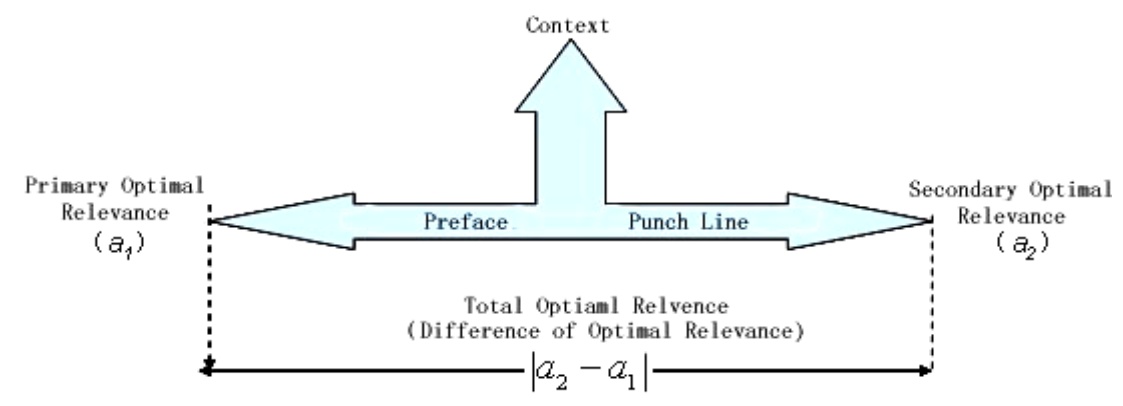

Figure 2. Relevance of Verbal Humor According to RT Inference 
As shown in the above figure, conventional RT inference from the preface and unconventional RT inference from the punch line all yield optimal relevance, however, the addressor trickily intrigued the story in a particular context, where his intentional misleading in the preface diverts the first conventional inference from the correct destination. As the direction of the first inference is opposite to the direction of the second inference, more optimal relevance obtained from the first inference and the second inference, less the total optimal relevance arrives. The addressee's confusion lies here: the punch line seems irrelevant to the preface, but the whole story matches well and is perfectly relevant. Then the contradiction between humor inference in the process of resolution and conventional inference is resolved.

3. A: If 2 is company and 3 is a crowd, what are 4 and 5 ?

B: The answer is 9. (Brain Teaser)

Analysis:

Meta-incongruitum $\left(n_{0}\right)$ : the paradox of semantic meaning of "and"

Preface: If 2 (-person) is company and 3 (-person) is a crowd

First conventional inference $\left(r_{0}\right)$

It's a word game (Implicated premise)

2 (-person) is company and 3 (-person) is a crowd (Explicated premise)

The answer should play a word game for " 4 " and " 5 " individually (Implicated conclusion)

Punch line: The answer is 9

Second resolution inference $\left(r_{1}\right)$

It's a mathematic exercise. (Implicated premise)

4 plus 5 (Explicated premise)

4 plus 5 makes 9 (Implicated conclusion)

The trick of this brain teaser composed of the meta-incongruitum lies in the pun of semantic meaning of word "and": the first "and": conj; connecting two or more phrases of similar function; the second "and" means addition). By playing the word game of "and", the author succeeds cheating us: logically, the preface is relevant to synonyms of some numbers, so the answer should be relevant to synonyms of some more numbers, and a simplest addition exercise is disguised to a capricious word game, which misleads the hearer to choose the first semantic meaning of "and".

Now the humorousness of this brain teaser is measurable:

Where there is one incongruitum, the paradox of semantic meaning of "and", so $n_{0}=1$, and there is one resolution inference, so $r_{1}=1$;

Humorousness $=$ incongruitum + resolutor $=n_{0}+r_{l}=1+1=2$.

Verb humors with a single incongruitum and a single resolutor are widely seen in word game and brain teaser because most of them require the addressee to make response in no time, otherwise the little fun will diminish as the addressee spends much time obtaining an absurd answer.

The above example shows the relevance of the punch line with the whole text, and accordingly Table I is modified as follows:

TABLE 2

CONVERSION TABLE OF $1+1$ TWO FACTOR FORMULAE (REVISED)

\begin{tabular}{|l|l|l|l|l|l|l|l|}
\hline $\begin{array}{l}\text { Incongruitum } \\
(x \text { Variable })\end{array}$ & $\begin{array}{l}\text { Resolutor } \\
(y \text { Variable })\end{array}$ & $\begin{array}{l}\text { Humorousness } \\
\text { (Sum })\end{array}$ & $\begin{array}{l}\text { Contextual } \\
\text { Effect }\end{array}$ & $\begin{array}{l}\text { Processing } \\
\text { Effort }\end{array}$ & \multicolumn{2}{|l|}{ Relevance } \\
\hline \multirow{2}{*}{$\geqq 1$} & $\geqq 2$ & Positive Growth & Ascend & $n_{0}$ & $n_{l}$ & $\begin{array}{c}\text { Sum } \\
\left|n_{0}-n_{l}\right|\end{array}$ \\
\cline { 4 - 8 } & & & Max & Max & Min \\
\hline
\end{tabular}

Note: neither incongruitum nor resolutor will disappear automatically whether ideal contextual effects are achieved or not, so neither $x$ nor $y$ will be a negative number.

\section{B. Multiple Incongruitums and Resolutors}

One incongruitum plus one resolutor is the simplest form of verbal humor, which makes sense by first conventional inference and second resolution inference and almost accords with the basic $1+1=2$ equation. However, most jokes or loose talk are no easy job, requiring the addressee's effort to deal with multiple incongruitums ( $\geqq 2$ ) and resolutors $(\geqq 2)$. From a single factor to multiple factors, those new incongruitums or resolutors are not the addresser's invention, but mostly, the result of motivated adjustment of P3, which forces the addressee to take those changes for granted and reconsider the original context where the meta-incongruitum survives. Hence the addressee has to make a second or a third resolution inference during the process of reconsideration, new resolutors $r_{1}, r_{2}, r_{3} \ldots$ are generated. At the same time, the addressee will bear a new expected value in mind during the second or a third resolution inference, and this new expected value strays further from the real destination, which means generation of one or more than one neoincongruitums $\left(n_{1}, n_{2}, n_{3} \ldots\right)$. The famous "counter-dilemma" case Protagoras vs. Euathlus (Suber, 1990) is one of the best examples.

Protagoras taught rhetoric and argumentation, which in ancient Greece comprised the education of a lawyer. Euathlus wished to learn these arts and asked Protagoras to teach him. He said he could not pay right away but promised to pay in full after he won his first case. Protagoras agreed, and taught Euathlus rhetoric and argumentation. Some accounts 
say Protagoras sued Euathlus for payment immediately upon the completion of the lessons; other accounts say he waited until it was evident that Euathlus was not taking on any cases. The case was heard in the court of Areopagus in Athens.

The judge asked Protagoras why he thought he had a claim against Euathlus. Protagoras argued, "I will either win this case or lose it. If I win it, then Euathlus must pay me, by the judgment of the court. If I lose it, then he must pay me, under our contract. So he must pay me either way."

(To be continued)

Call it here, the story has already made verbal humor because:

Meta-incongruitum $\left(\boldsymbol{n}_{\boldsymbol{0}}\right)$ : the dilemma of Euathlus to pay his debts whether he wins the case or lose it.

Preface: If I win it, then Euathlus must pay me, by the judgment of the court.

First conventional inference $\left(r_{0}\right)$

In a court, the plaintiff either wins or loses the case. (Implicated premise)

Protagoras wins the case. (Explicated premise)

The judge will ordain Euathlus to pay his debts. (Implicated conclusion)

Punch line: If I lose it, then he must pay me, under our contract. So he must pay me either way

Second resolution inference $\left(r_{l}\right)$

In a court, the plaintiff either wins or loses the case. (Implicated premise)

Protagoras loses the case. (Explicated premise)

Euathlus shall pay Protagoras on his promise. (Implicated conclusion)

By calculating all the favorable conditions in the court and of the contract, Protagoras had a "wily scheme" in bringing the suit in the first place (probably to win in a second suit if he lost the first). His words are witty and appreciating, so both the judge and the audience are impressed. Humorousness then is calculating as meta-incongruitum + initial resolutor $=n_{0}+r_{1}=1+1=2$.

However, the court, as said, wouldn't feel so puzzled that it adjourned for 100 years if the case is over like this. Actually when all of it suggests that Protagoras should have won, Euathlus showed off his cleverness to make things totally different, please see Continue.

(Continue)

"I will either win this case or lose it. If I win it, then Euathlus must pay me, by the judgment of the court. If I lose it, then he must pay me, under our contract. So he must pay me either way."

The judge was impressed, and asked Euathlus to reply. Euathlus had learned his lessons well, and replied, "I too will either win this case or lose it. If I win it, then I need not pay Protagoras, by the judgment of the court. If I lose it, then I need not pay him, under our contract. So I need not pay him either way."

Euathlus's response deserves separate, special treatment as a classical illustration of the use of reflexive language for responding a "dilemma" with a "counter-dilemma". Reflexivity is the generic term for all types of logical circularity: the self-reference of signs, the self-application of principles, the self-justification and self-refutation of propositions and inferences, the self-creation and self-destruction of legal and logical entities, the self-limitation and self-augmentation of powers, circular reasoning, circular causation, vicious and benign circles, and feedback systems. The common practice of using self-reference to cover all this territory is inaccurate and unfortunate. (Suber, 1990)

Euathlus would be better off hiring a lawyer because, if he won with a lawyer, his victory would be non-paradoxical, and if he lost with a lawyer, he would not yet have won his first case, however, it is by the price of losing humorousness of the story. Protagoras's argumentation has already formed a verbal humor. But according to our questionnaire, most participants agree that the essence of this story lie in the latter part of Euathlus's retort, a proof that the latter part is more humorous than the former part, i.e. with more amount of humorousness:

\section{Analysis:}

Neo-incongruitum $\left(\boldsymbol{n}_{1}\right)$ : the dilemma of Protagoras to pay his debts whether he wins the case or lose it

Preface: If I win it, then I need not pay Protagoras, by the judgment of the court

\section{First conventional inference $\left(r_{0}\right)$}

In a court, the plaintiff either wins or loses the case. (Implicated premise)

Euathlus wins the case. (Explicated premise)

The judge will release Euathlus from his debts. (Implicated conclusion)

Punch line: If I lose it, then I need not pay him, under our contract. So I need not pay him either way.

\section{Second resolution inference $\left(r_{2}\right)$}

In a court, the plaintiff either wins or loses the case. (Implicated premise)

Euathlus loses the case. (Explicated premise)

Euathlus needn't pay Protagoras. (Implicated conclusion)

To sum up, this case contains two variable contextual factors, court and contract, clutching each other to form a dynamic circle. Both Protagoras and Euathlus found out proper and favorable conditions in this circle to defend themselves. The role of the one who takes the initiative is replaced as the contextual circle rolling, rendering Protagoras to lose his advantages at first. Because all of us as the audience of the story expect that Protagoras will be paid, and find wit and fun in Protagoras's words, the final counterturn is totally our expectation and consequently, the amazement 
brought by Euathlus's malicious prosecution surpasses the appreciation from Protegra. The evidence is the ascending humorousness as follows:

Humorousness $=$ meta-incongruitum + initial resolutor + neo-incongruitum $+2^{\text {nd }}$ resolutor

$$
=n_{0}+r_{1}+n_{2}+r_{2}=1+1+1+1
$$

$=4$.

Now simple humor including both single factors and multiple factors verify the validity of $1+1$ Two Factor Formulae formed on data collected from 300 questionnaires. A safe conclusion can be drawn that humorousness of verbal humor is the result of interaction between both incongruitums and resolutors under the common influence of three major parameters, cognitive capacity, laughing index and context. Variation of any parameter will lead to corresponding changes for two factors, followed by the change of humorousness accordingly.

\section{CONCLUSION}

\section{A. Findings}

This thesis aims at exploring the pragmatic mechanisms of interpretation of verbal humor, that is, to investigate why some of the utterances are perceived to be humorous and formulation of a trial model for measurement of humorousness accordingly, which will be deductively processed by setting $1+1$ Two Factor formulae based on our hypothesis and verification of the formulate by case study.

For the first target, many similar efforts, past and present, have been examined from different angles such as psychological, linguistic, pragmatic and cognitive. However, varied humor theories so far still remain sporadic and fragmentary. Humor scholars are mostly amateurs engaged in the pastime of musing about laughter. What is more, even few of them have concerned themselves with the cognition of the production of humor interpretation. Thus from the perspective of cognition, we have built a bridge with relevance to incongruity-resolution theory and harvested the following findings:

- RT combined with incongruity-resolution has a powerful theoretic force to explain the phenomenon of verbal humor. RT's inferential-ostensive communication is applicable to the verbal humor interpretation.

- Humor interpretation is guided by the Relevance Principle and it is a process of the hearer's search for (cognitive) maximal and (communicative) optimal relevance.

- According to RT, humor understanding is a perception and/or resolution of the incongruity from two different interpretations.

- Verbal humor must be studied within the context and the humor interpretation is also a process of contextual assumptions.

And as for the second target, humorousness, though literally understandable, is still a virgin land full of riddles. Based on our observation and our findings of pragmatic and cognitive mechanisms of verbal humor, we have further proposed a hypothesis and built a trial model for measurement of humorousness, which is verified by case study and data collected from 300 replies of our specially designed questionnaire, including:

- Humorousness is an indicator to test the quality of comic text, which is reflected by the contextual effects the addressee obtains after interpretation with processing efforts and the degree of the perlocutionary force of laughter and surprise.

- Humorousness, the degree of humor, is not a scattered random sequence that can be qualitatively defined, but a continuum measurable and adjustable through a set of pragmatic devices and strategies.

- Humorousness can be defined as the humorous effect of a discourse or utterance in special context, but the changing rules of humorousness differ from general discourses or utterances in daily communication in terms of relevance.

- Humorousness varies along the degree of relevance at different intervals. Such intervals mainly depend on one's cognitive ability and are assisted by context.

- Humorousness is the result of interaction between both factors of incongruitum and resolutors under the common influence of three major parameters, cognitive capacity, laughing index and context. Variation of any parameter will lead to corresponding changes for two factors, followed by ascending or descending of humorousness accordingly.

\section{B. Contributions}

The model succeeds in deconstructing humor into two factors: incongruitum and resolutor and three parameters: cognitive capacity, laughing index and context. Bearing this in mind, a composer can have a clear clue for design of his work and improvement of humor quality. Cognitive capacity will help the composer choose his audience, avoiding misinterpretation of his punch line, which leads to communication failure. Laughing index tells the composer about what makes the audience laugh, so both incongruity and resolution will be considered in his composition. Meanwhile, another technique the composer should learn is to add incongruitum and resolutor for purpose of improving quality of his verbal humor. Knowledge of context is especially useful in renewal of an old joke. As far as we know, many old stories rejuvenate after being set in another context. For example, the American Film Monster Shriek mainly succeeded in that an ugly monster replaced the handsome prince in legend Cinderella. In a word, a skillful composer may provide 
the audience with a story funny enough to their satisfaction by delicate design of two factors and three major parameters of verbal humor in his composition because he knows well what the audience want and how to make them laugh.

In the long run, research on humorousness will open a new horizon for humor research and help integration of humor theories and analysis of various disciplines. Moreover, consciousness of humorousness will help ordinary people improve their sense of humor and ability to appreciate funny things in life.

\section{REFERENCES}

[1] Hou Guojin. (2004). Computing Indirectness of Language. Journal of Sichuan International Studies University, 6, 66-72.

[2] Liu Naishi. (2005). Incongruity and Resolution in Humor from the Perspective of Relative Theory. Journal of PLA Institute of Foreign Languages, 1, 16-19.

[3] Liu Ping. (2005). Verbal Humor in Linguistic Studies. Hubei Social Science, 5, 101-103.

[4] Palmer. (1994). Taking Humor Seriously. London and New York: Routledge.

[5] Suber, P. (1990). The paradox of self-amendment. http://legacy.earlham.edu/ peters/writing/psa/index.htm (accessed 04/1/2013).

[6] Wang Yong. (2002). Smile Mechanism of Humorous Speech. Social Sciences in Nanjing, 2, 65-67.

[7] William J. Pepicello, Robert W. Weisberg. (1983). Linguistics and Humor. In Paul E. McGhee and Jeffrey H. Goldstein (Ed.), Handbook of Humor Research: Basic issues. New York: Springer-Verlag, 59-83.

[8] Xu Lixin. (2004). A Study on Humorous Discourse. Kaifeng: Henan University Press.

Wanfang Zhang earned his MA in Translation Theory and Practice from Ningbo University, China, in 2006. He is currently a lecturer in the School of Foreign Languages, Wuhan Polytechnic University, Wuhan, China. His research interests cover translation studies, cultural studies, intercultural communication and cognitive linguistics. 